\title{
Further Experimental Study on the Short=timed Peroxidase Reaction of Blood Polynuclears of Rice=diseased Mice. Comparison between Absolute and Relative $B *$ =avitaminosis and $B=$ avitaminosis with Insufficiency of Liver Function.
}

104th Report of the Peroxidase Reaction.

By

Tamotsu Suzuki.

(鈴 木 保)

(From the Department of Pediatrics, Faculty of Medicine, Tohoku Imperial University, Sendai. Director: Prof. A. Sato.)

\section{Introduction.}

In the 55th 1 Report of the Peroxidase Reaction I described 1. that the shortest peroxidase stain-time of blood polynuclears (by copper method ${ }^{2)}$ becomes prolonged as a symptom of rice disease, and so much the more prolonged as it becomes severe, 2 . that the prolongation appears earlier than other symptoms of rice disease, and 3 . that this phenomenon is due to B-deficit. Further it was suggested that a certain substance or substances are produced in a condition of B-deficit and makes the peroxidase reaction weaker.

In 1925, Ogata and his collaboraters ${ }^{3}$ reported their result that the rice disease of pigeons fed on absolutely B-deficient food and that of pigeons fed on relatively B-deficient food are identical, and that in the latter only the period of incubation was longer than in the former.

\footnotetext{
* In the present paper $B_{1}$ is meant throughout the paper.

1) T. Suzuki, Tohoku J. Exp. Med., 1935, 25, 201.

2) A. Sato and S. Sekiya, Tohoku J. Exp. Med., 1926, 7, 111. (Also Cf. A. Sato and T. Suzuki, Tohoku, J. Exp. Med., 1936, 29, 92.)

3) T. Ogata, S. Kawasaki, S. Suzuki and S. Kagosima, Mitteilungen aus der Medizinischen Fakultät der Kaiserlichen Universität zu Tokyo, 1925, 32, 431.
} 
The present paper is a study performed from the point of shorttimed peroxidase reaction of blood polynuclears, on a difference between absolutely* and relatively B-deficient feeding and on the influence of an insufficiency of liver function upon B-avitaminosis.

\section{Method of Experiment.}

1. Feeding. Mice were fed on polished and thoroughly washed rice in experiments of the first and the third series, but mice in experiments of the second series were fed on polished rice, which had not been washed at all. The unwashed polished rice has of course a small amount of rice-bran, so that feeding on unwashed polished rice is a relative B-deficit. For mice in experiments of the fourth series, unpolished wheat, which is sufficient in vitamin $B$, was used in order to keep them from B-avitaminosis. All the animals were left to themselves to eat rice or wheat, as the case might be, and to drink water.

2. Injection of $3 \%$ ammonium chloride. In experiments of the third and fourth series, mice were injected subcutaneously every second day with $3 \%$ ammonium chloride, the amount of which was 0.01 c.c. per grm. of body weight for each mouse.

3. Blood examination. Blood smears were made from flowing blood from the cut end of the tail, and stained by the short-timed peroxidase method. ${ }^{4}$ Normal neutrophile leucocytes of mice ought to be stained perceptibly in 2 seconds. ${ }^{1)}$ Those smears which were not stained in 2 seconds' time-the smears with a prolongation-iwere treated with ether for twenty seconds and stained one after another with longer times of staining, until they became just perceptibly stained.

\section{Result of Experiment.}

1. The first series of experiment (Cf. Table 1).

Mice were fed on thoroughly washed polished rice, so that these mice were cases of absolute B-deficit. The result is tabulated as will be seen in Table 1 (Cf. No. 1-No. 7 in Table 1).

* This word is conveniently used in contrast to the word "relatively" (Cf. Method of Experiment).

4) K. Suzuki, Tohoku J. Exp. Med., 1932, 19, 262 and T. Suzuki, Tohoku J. Exp.Med., 1934, 23, 23. (Also Cf. A. S a to and T. S u z u ki, Tohoku J. Exp. Med., 1936, 29, 92.) 
The length of life of mice after the commencement of feeding with absolutely B-deficient food was 21.4 days on an average. A prolongation of the shortest peroxidase stain-time occurred after averaging 5.4

\section{Table 1.}

The short-timed peroxidase reaction of blood leucocytes and symptoms of mice in the case of feeding with thoroughly washed polished rice.

No. 1

(No. 1-No. 7)

\begin{tabular}{|c|c|c|c|c|c|}
\hline \multirow{2}{*}{\multicolumn{2}{|c|}{$\begin{array}{c}\text { Day of experiment } \\
\text { (Date) }\end{array}$}} & \multirow{2}{*}{$\begin{array}{l}\text { Body } \\
\text { weight in } \\
\text { grms. }\end{array}$} & \multicolumn{2}{|c|}{$\begin{array}{l}\text { Shortest peroxidase } \\
\text { stain-time }\end{array}$} & \multirow[b]{2}{*}{ Remarks } \\
\hline & & & Fresh film & $\begin{array}{c}\text { After } \\
\text { treating* } \\
\text { with ether }\end{array}$ & \\
\hline \multicolumn{2}{|c|}{$\begin{array}{c}\text { Before the exp. } \\
(13 / I I)\end{array}$} & 17.5 & $2^{\prime \prime}$ & & Pregnancy \\
\hline $\begin{array}{c}\text { Days } \\
\text { of } \\
\text { feeding }\end{array}$ & $\begin{array}{l}2(15 . \mathrm{II}) \\
4 \\
6 \\
8 \\
10 \\
12 \\
14 \\
16 \\
18 \\
20 \\
22 \\
24\end{array}$ & $\begin{array}{l}19.0 \\
20.5 \\
21.0 \\
15.5 \\
15.0 \\
14.5 \\
11.5 \\
11.5 \\
11.5 \\
12.0 \\
11.5 \\
10.0\end{array}$ & $\begin{array}{r}2^{\prime \prime} \\
3^{\prime \prime} \\
3^{\prime \prime} \\
3^{\prime \prime} \\
4^{\prime \prime} \\
6^{\prime \prime} \\
7^{\prime \prime} \\
8^{\prime \prime} \\
10^{\prime \prime} \\
10^{\prime \prime} \\
10^{\prime \prime} \\
11^{\prime \prime}\end{array}$ & $\begin{array}{l}2^{\prime \prime} \\
2^{\prime \prime} \\
2^{\prime \prime} \\
2^{\prime \prime} \\
2^{\prime \prime} \\
2^{\prime \prime} \\
3^{\prime \prime} \\
4^{\prime \prime} \\
5^{\prime \prime} \\
5^{\prime \prime} \\
5^{\prime \prime}\end{array}$ & $\begin{array}{l}\text { On the } 7 \text { th day abor- } \\
\text { tion } \\
\text { Diarrhoea } \\
\text { Paresis } \\
\text { Paresis increased } \\
\text { Gait impossible, death } \\
\text { in the afternoon }\end{array}$ \\
\hline
\end{tabular}

* The shortest peroxidase stain-time of leucocytes on a film which had been treated with ether.

No. 2

\begin{tabular}{|c|c|c|c|c|c|}
\hline \multirow{2}{*}{\multicolumn{2}{|c|}{$\begin{array}{c}\text { Day of experiment } \\
\text { (Date) }\end{array}$}} & \multirow[b]{2}{*}{$\begin{array}{l}\text { Body } \\
\text { weight in } \\
\text { grms. }\end{array}$} & \multicolumn{2}{|c|}{$\begin{array}{l}\text { Shortest peroxidase } \\
\text { stain-time }\end{array}$} & \multirow[b]{2}{*}{ Remarks } \\
\hline & & & Fresh film & $\begin{array}{c}\text { After } \\
\text { treating* } \\
\text { with ether }\end{array}$ & \\
\hline \multicolumn{2}{|c|}{$\begin{array}{c}\text { Before the exp. } \\
(13 / \mathrm{II})\end{array}$} & 15.0 & $2^{\prime \prime}$ & & \\
\hline $\begin{array}{c}\text { Days } \\
\text { of } \\
\text { feeding }\end{array}$ & $\begin{array}{r}2(15 . \mathrm{II}) \\
4 \\
6 \\
8 \\
10 \\
12 \\
14 \\
16 \\
18 \\
19\end{array}$ & $\begin{array}{l}14.0 \\
15.0 \\
14.5 \\
13.5 \\
14.0 \\
13.0 \\
11.5 \\
11.0 \\
10.5\end{array}$ & $\begin{array}{l}2^{\prime \prime} \\
2^{\prime \prime} \\
3^{\prime \prime} \\
3^{\prime \prime} \\
3^{\prime \prime} \\
4^{\prime \prime} \\
6^{\prime \prime} \\
7^{\prime \prime} \\
8^{\prime \prime}\end{array}$ & $\begin{array}{l}2^{\prime \prime} \\
2^{\prime \prime} \\
2^{\prime \prime} \\
2^{\prime \prime} \\
2^{\prime \prime} \\
3^{\prime \prime} \\
3^{\prime \prime}\end{array}$ & $\begin{array}{l}\text { Diarrhoea } \\
\text { Paresis } \\
\text { Death }\end{array}$ \\
\hline
\end{tabular}

* Cf. the foot note to No. 1. 
days of feeding, generally much earlier than any clinical symptom. And when a clinical symptom manifested itself at all, the prolongation in question was as remarkably long as 6 or 7 seconds. After treating the blood specimens with ether, the shortest peroxidase stain-time became shorter than that of fresh blood films. Thus, the result of Table 1 remains just the same as has been shown in a preceding paper of my own (Cf. the 55th Report of the peroxidase Reaction).

No. 3

\begin{tabular}{|c|c|c|c|c|c|}
\hline \multirow{2}{*}{\multicolumn{2}{|c|}{$\begin{array}{c}\text { Day of experiment } \\
\text { (Date) }\end{array}$}} & \multirow{2}{*}{$\begin{array}{l}\text { Body } \\
\text { weight in } \\
\text { grms. }\end{array}$} & \multicolumn{2}{|c|}{$\begin{array}{l}\text { Shortest peroxidase } \\
\text { stain-time }\end{array}$} & \multirow[b]{2}{*}{ Remarks } \\
\hline & & & Fresh film & $\begin{array}{c}\text { After } \\
\text { treating* } \\
\text { with ether }\end{array}$ & \\
\hline \multicolumn{2}{|c|}{$\begin{array}{c}\text { Before the exp. } \\
(13 / \mathbf{I I})\end{array}$} & 15.5 & $2^{\prime \prime}$ & & \\
\hline $\begin{array}{l}\text { Days } \\
\text { of } \\
\text { feeding }\end{array}$ & $\begin{array}{r}2(15 . \mathrm{II}) \\
4 \\
6 \\
8 \\
10 \\
12 \\
14 \\
16 \\
18 \\
19\end{array}$ & $\begin{array}{l}15.0 \\
15.0 \\
15.0 \\
14.5 \\
14.0 \\
12.5 \\
11.0 \\
10.5 \\
10.0\end{array}$ & $\begin{array}{l}2^{\prime \prime} \\
2^{\prime \prime} \\
3^{\prime \prime} \\
3^{\prime \prime} \\
3^{\prime \prime} \\
4^{\prime \prime} \\
6^{\prime \prime} \\
7^{\prime \prime} \\
9^{\prime \prime}\end{array}$ & $\begin{array}{l}\mathbf{2}^{\prime \prime} \\
2^{\prime \prime} \\
2^{\prime \prime} \\
2^{\prime \prime} \\
2^{\prime \prime} \\
2^{\prime \prime} \\
4^{\prime \prime}\end{array}$ & $\begin{array}{l}\text { Diarrhoea } \\
\text { Paresis } \\
\text { Convulsion } \\
\text { Death }\end{array}$ \\
\hline
\end{tabular}

* Cf. the foot note to No. 1.

No. 4

\begin{tabular}{|c|c|c|c|c|c|}
\hline \multirow{2}{*}{\multicolumn{2}{|c|}{$\begin{array}{c}\text { Day of experiment } \\
\text { (Date) }\end{array}$}} & \multirow{2}{*}{$\begin{array}{l}\text { Body } \\
\text { weight in } \\
\text { grms. }\end{array}$} & \multicolumn{2}{|c|}{$\begin{array}{l}\text { Shortest peroxidase } \\
\text { stain-time }\end{array}$} & \multirow[b]{2}{*}{ Remarks } \\
\hline & & & Fresh film & $\begin{array}{c}\text { After } \\
\text { treating* } \\
\text { with ether }\end{array}$ & \\
\hline \multicolumn{2}{|c|}{$\begin{array}{c}\text { Before the exp. } \\
(11 / \text { III })\end{array}$} & 18.0 & $2^{\prime \prime}$ & & \\
\hline $\begin{array}{c}\text { Days } \\
\text { of } \\
\text { feeding }\end{array}$ & $\begin{array}{r}2(13 . \mathrm{III}) \\
4 \\
6 \\
8 \\
10 \\
12 \\
14 \\
16 \\
18 \\
20 \\
22\end{array}$ & $\begin{array}{l}18.0 \\
18.0 \\
16.0 \\
17.0 \\
15.5 \\
14.5 \\
14.0 \\
12.5 \\
12.5 \\
12.0 \\
10.5\end{array}$ & $\begin{array}{l}2^{\prime \prime} \\
2^{\prime \prime} \\
3^{\prime \prime} \\
3^{\prime \prime} \\
4^{\prime \prime} \\
4^{\prime \prime} \\
4^{\prime \prime} \\
5^{\prime \prime} \\
6^{\prime \prime} \\
7^{\prime \prime} \\
7^{\prime \prime}\end{array}$ & $\begin{array}{l}2^{\prime \prime} \\
2^{\prime \prime} \\
2^{\prime \prime} \\
2^{\prime \prime} \\
2^{\prime \prime} \\
2^{\prime \prime} \\
2^{\prime \prime} \\
3^{\prime \prime} \\
3^{\prime \prime}\end{array}$ & $\begin{array}{l}\text { Paresis } \\
\text { Gait impossible, death } \\
\text { in the evening }\end{array}$ \\
\hline
\end{tabular}

* Cf. the foot note to No. 1 . 
No. 5

\begin{tabular}{|c|c|c|c|c|c|}
\hline \multirow{2}{*}{\multicolumn{2}{|c|}{$\begin{array}{c}\text { Day of experiment } \\
\text { (Date) }\end{array}$}} & \multirow{2}{*}{$\begin{array}{l}\text { Body } \\
\text { weight in } \\
\text { grms. }\end{array}$} & \multicolumn{2}{|c|}{$\begin{array}{c}\text { Shortest peroxidase } \\
\text { stain-time }\end{array}$} & \multirow[b]{2}{*}{ Remarks } \\
\hline & & & Fresh film & $\begin{array}{c}\text { After } \\
\text { treating* } \\
\text { with ether }\end{array}$ & \\
\hline \multicolumn{2}{|c|}{$\begin{array}{l}\text { Before the exp. } \\
\text { (11/III) }\end{array}$} & 20.0 & $2^{\prime \prime}$ & & \\
\hline $\begin{array}{c}\text { Days } \\
\text { of } \\
\text { feeding }\end{array}$ & $\begin{array}{l}2(13 . \mathrm{III}) \\
4 \\
6 \\
8 \\
10 \\
12 \\
14 \\
16 \\
18 \\
20 \\
22 \\
23\end{array}$ & $\begin{array}{l}20.5 \\
21.0 \\
21.0 \\
20.0 \\
20.5 \\
20.0 \\
19.0 \\
18.5 \\
17.5 \\
17.0 \\
14.0\end{array}$ & $\begin{array}{l}2^{\prime \prime} \\
2^{\prime \prime} \\
3^{\prime \prime} \\
3^{\prime \prime} \\
4^{\prime \prime} \\
5^{\prime \prime} \\
5^{\prime \prime} \\
5^{\prime \prime} \\
6^{\prime \prime} \\
7^{\prime \prime} \\
7^{\prime \prime}\end{array}$ & $\begin{array}{c}2^{\prime \prime} \\
2^{\prime \prime} \\
2^{\prime \prime} \\
2^{\prime \prime} \\
2^{\prime \prime} \\
2^{\prime \prime} \\
2^{\prime \prime} \\
3^{\prime \prime} \\
3^{\prime \prime}\end{array}$ & $\begin{array}{l}\text { Paresis } \\
\text { Diarrhoea, gait diff- } \\
\text { cult } \\
\text { Death }\end{array}$ \\
\hline
\end{tabular}

* Cf. the foot note to No. 1 .

No. 6

\begin{tabular}{|c|c|c|c|c|c|}
\hline \multirow{2}{*}{\multicolumn{2}{|c|}{$\begin{array}{c}\text { Day of experiment } \\
\text { (Date) }\end{array}$}} & \multirow{2}{*}{$\begin{array}{l}\text { Body } \\
\text { weight in } \\
\text { grms. }\end{array}$} & \multicolumn{2}{|c|}{$\begin{array}{l}\text { Shortest peroxidase } \\
\text { stain-time }\end{array}$} & \multirow[b]{2}{*}{ Remarks } \\
\hline & & & Fresh film & $\begin{array}{c}\text { After } \\
\text { treating* } \\
\text { with ether }\end{array}$ & \\
\hline \multicolumn{2}{|c|}{$\begin{array}{c}\text { Before the exp. } \\
(4 / \mathrm{IV})\end{array}$} & 12.0 & $2^{\prime \prime}$ & & \\
\hline $\begin{array}{c}\text { Days } \\
\text { of } \\
\text { feeding }\end{array}$ & $\begin{array}{l}2(6 . \mathrm{IV}) \\
4 \\
6 \\
8 \\
10 \\
12 \\
14 \\
16 \\
18 \\
20 \\
22\end{array}$ & $\begin{array}{r}11.5 \\
11.5 \\
11.0 \\
11.5 \\
10.5 \\
9.5 \\
9.5 \\
9.0 \\
8.5 \\
9.0 \\
8.5\end{array}$ & $\begin{array}{l}2^{\prime \prime} \\
2^{\prime \prime} \\
3^{\prime \prime} \\
3^{\prime \prime} \\
4^{\prime \prime} \\
5^{\prime \prime} \\
5^{\prime \prime} \\
6^{\prime \prime} \\
6^{\prime \prime} \\
7^{\prime \prime} \\
8^{\prime \prime}\end{array}$ & $\begin{array}{l}2^{\prime \prime} \\
2^{\prime \prime} \\
2^{\prime \prime} \\
2^{\prime \prime} \\
2^{\prime \prime} \\
2^{\prime \prime} \\
2^{\prime \prime} \\
3^{\prime \prime} \\
3^{\prime \prime}\end{array}$ & $\begin{array}{l}\text { Paresis } \\
\text { Gait impossible, death } \\
\text { in the evening }\end{array}$ \\
\hline
\end{tabular}

* Cf. the foot note to No. 1.

2. The second series of experiment (Cf. Table 2).

Mice were fed on unwashed polished rice, and thus in a state of relative B-avitaminosis. The result of investigation is as shown in Table 2 (Cf. No. 8-No. 13 in Table 2).

The length of life of mice after the commencement of feeding: with relatively B-deficient food was 36 days on an average. A prolongation of the shortest peroxidase stain-time occurred after averag- 
No. 7

\begin{tabular}{|c|c|c|c|c|c|}
\hline \multirow{2}{*}{\multicolumn{2}{|c|}{$\begin{array}{c}\text { Day of experiment } \\
\text { (Date) }\end{array}$}} & \multirow{2}{*}{$\begin{array}{c}\text { Body } \\
\text { weight in } \\
\text { grms. }\end{array}$} & \multicolumn{2}{|c|}{$\begin{array}{l}\text { Shortest peroxidase } \\
\text { stain-time }\end{array}$} & \multirow[b]{2}{*}{ Remarks } \\
\hline & & & Fresh film & $\begin{array}{c}\text { After } \\
\text { treating* } \\
\text { with ether }\end{array}$ & \\
\hline \multicolumn{2}{|c|}{$\begin{array}{l}\text { Before the exp. } \\
(4 / \mathrm{IV})\end{array}$} & 11.0 & $2^{\prime \prime}$ & & \\
\hline $\begin{array}{l}\text { Days } \\
\text { of } \\
\text { feeding }\end{array}$ & $\begin{array}{l}2(6 . I V) \\
4 \\
6 \\
8 \\
10 \\
12 \\
14 \\
16 \\
18 \\
20 \\
21\end{array}$ & $\begin{array}{r}10.5 \\
10.5 \\
10.5 \\
9.0 \\
9.0 \\
9.0 \\
8.5 \\
8.5 \\
7.5 \\
7.0 \\
6.5\end{array}$ & $\begin{array}{l}2^{\prime \prime} \\
3^{\prime \prime} \\
3^{\prime \prime} \\
4^{\prime \prime} \\
4^{\prime \prime} \\
5^{\prime \prime} \\
5^{\prime \prime} \\
6^{\prime \prime} \\
7^{\prime \prime} \\
7^{\prime \prime} \\
9^{\prime \prime}\end{array}$ & $\begin{array}{l}2^{\prime \prime} \\
2^{\prime \prime} \\
2^{\prime \prime} \\
2^{\prime \prime} \\
2^{\prime \prime} \\
2^{\prime \prime} \\
2^{\prime \prime} \\
2^{\prime \prime} \\
3^{\prime \prime} \\
3^{\prime \prime}\end{array}$ & $\begin{array}{l}\text { Paresis } \\
\text { Gait difficult, death } \\
\text { in the night }\end{array}$ \\
\hline
\end{tabular}

* Cf, the foot note to No. 1.

TABLe 2.

The short-timed peroxidase reaction of blood leucocytes and symptoms of mice in the case of feeding with not washed polished rice.

No. 8

(No.8-No. 13)

\begin{tabular}{|c|c|c|c|c|c|}
\hline \multirow{2}{*}{\multicolumn{2}{|c|}{$\begin{array}{c}\text { Day of experiment } \\
\text { (Date) }\end{array}$}} & \multirow{2}{*}{$\begin{array}{l}\text { Body } \\
\text { weight in } \\
\text { grms. }\end{array}$} & \multicolumn{2}{|c|}{$\begin{array}{c}\text { Shortest peroxidase } \\
\text { stain-time }\end{array}$} & \multirow[b]{2}{*}{ Remarks } \\
\hline & & & Fresh film & $\begin{array}{c}\text { After } \\
\text { treating* } \\
\text { with ether }\end{array}$ & \\
\hline \multicolumn{2}{|c|}{$\begin{array}{c}\text { Before the exp. } \\
(19 / \mathrm{II})\end{array}$} & 13.0 & $2^{\prime \prime}$ & & \\
\hline $\begin{array}{c}\text { Days } \\
\text { of } \\
\text { feeding }\end{array}$ & $\begin{array}{l}2(21 . \mathrm{II}) \\
4 \\
6 \\
8 \\
10 \\
12 \\
14 \\
16 \\
18 \\
20 \\
22 \\
24 \\
26 \\
28 \\
30 \\
32 \\
34\end{array}$ & $\begin{array}{l}13.5 \\
14.0 \\
13.0 \\
12.5 \\
12.0 \\
11.0 \\
10.5 \\
10.0 \\
10.5 \\
11.0 \\
11.0 \\
10.0 \\
10.0 \\
10.5 \\
10.0 \\
10.5 \\
10.0\end{array}$ & $\begin{array}{l}2^{\prime \prime} \\
2^{\prime \prime} \\
2^{\prime \prime} \\
2^{\prime \prime} \\
2^{\prime \prime} \\
3^{\prime \prime} \\
3^{\prime \prime} \\
3^{\prime \prime} \\
3^{\prime \prime} \\
3^{\prime \prime} \\
4^{\prime \prime} \\
4^{\prime \prime} \\
4^{\prime \prime} \\
4^{\prime \prime} \\
4^{\prime \prime} \\
5^{\prime \prime} \\
6^{\prime \prime}\end{array}$ & $\begin{array}{l}2^{\prime \prime} \\
2^{\prime \prime} \\
2^{\prime \prime} \\
2^{\prime \prime} \\
2^{\prime \prime} \\
2^{\prime \prime} \\
2^{\prime \prime} \\
2^{\prime \prime} \\
2^{\prime \prime} \\
2^{\prime \prime} \\
2^{\prime \prime} \\
2^{\prime \prime}\end{array}$ & $\begin{array}{l}\text { Paresis } \\
\text { Paresis increased, } \\
\text { death in the evening }\end{array}$ \\
\hline
\end{tabular}

* The shortest peroxidase stain-time of leucocytes on a film which had been treated with ether. 
ing 11.3 days of feeding, much earlier than any clinical symptom. And when a clinical symptom manifested itself at all, then the prolongation

No. 9

\begin{tabular}{|c|c|c|c|c|c|}
\hline \multirow{2}{*}{\multicolumn{2}{|c|}{$\begin{array}{c}\text { Day of experiment } \\
\text { (Date) }\end{array}$}} & \multirow{2}{*}{$\begin{array}{l}\text { Body } \\
\text { weight in } \\
\text { grms. }\end{array}$} & \multicolumn{2}{|c|}{$\begin{array}{l}\text { Shortest peroxidase } \\
\text { stain-time }\end{array}$} & \multirow[b]{2}{*}{ Remarks } \\
\hline & & & Fresh film & $\begin{array}{c}\text { After } \\
\text { treating* } \\
\text { with ether }\end{array}$ & \\
\hline \multicolumn{2}{|c|}{$\begin{array}{c}\text { Before the exp. } \\
(19 / \mathrm{II})\end{array}$} & 12.5 & $2^{\prime \prime}$ & & \\
\hline $\begin{array}{c}\text { Days } \\
\text { of } \\
\text { feeding }\end{array}$ & $\begin{array}{l}2(21, \mathrm{II}) \\
4 \\
6 \\
8 \\
10 \\
12 \\
14 \\
16 \\
18 \\
20 \\
22 \\
24 \\
26 \\
28 \\
30\end{array}$ & \begin{tabular}{r|}
13.5 \\
13.0 \\
13.5 \\
13.5 \\
13.5 \\
13.5 \\
13.5 \\
13.0 \\
13.5 \\
13.5 \\
12.5 \\
12.0 \\
11.5 \\
10.5 \\
9.0
\end{tabular} & $\begin{array}{l}2^{\prime \prime} \\
2^{\prime \prime} \\
2^{\prime \prime} \\
2^{\prime \prime} \\
3^{\prime \prime} \\
3^{\prime \prime} \\
3^{\prime \prime} \\
4^{\prime \prime} \\
4^{\prime \prime} \\
4^{\prime \prime} \\
4^{\prime \prime} \\
4^{\prime \prime} \\
4^{\prime \prime} \\
4^{\prime \prime} \\
5^{\prime \prime}\end{array}$ & $\begin{array}{l}2^{\prime \prime} \\
2^{\prime \prime} \\
2^{\prime \prime} \\
2^{\prime \prime} \\
2^{\prime \prime} \\
2^{\prime \prime} \\
2^{\prime \prime} \\
2^{\prime \prime} \\
2^{\prime \prime} \\
2^{\prime \prime} \\
2^{\prime \prime}\end{array}$ & $\begin{array}{l}\text { Diarrhoea } \\
\text { Death in the morning }\end{array}$ \\
\hline
\end{tabular}

* Cf. the foot note to No. 8.

No. 10

\begin{tabular}{|c|c|c|c|c|c|}
\hline \multirow{2}{*}{\multicolumn{2}{|c|}{$\begin{array}{c}\text { Day of experiment } \\
\text { (Date) }\end{array}$}} & \multirow{2}{*}{$\begin{array}{l}\text { Body } \\
\text { weight in } \\
\text { grms. }\end{array}$} & \multicolumn{2}{|c|}{$\begin{array}{l}\text { Shortest peroxidase } \\
\text { stain-time }\end{array}$} & \multirow[b]{2}{*}{ Remarks } \\
\hline & & & Fresh film & $\begin{array}{c}\text { After } \\
\text { treating* } \\
\text { with ether }\end{array}$ & \\
\hline \multicolumn{2}{|c|}{$\begin{array}{c}\text { Before the exp, } \\
(19 / \mathrm{II})\end{array}$} & 10.0 & $2^{\prime \prime}$ & & \\
\hline $\begin{array}{c}\text { Days } \\
\text { of } \\
\text { feeding }\end{array}$ & $\begin{array}{l}2(21 . \mathrm{II}) \\
4 \\
6 \\
8 \\
10 \\
12 \\
14 \\
16 \\
18 \\
20 \\
22 \\
24 \\
26 \\
28 \\
30 \\
32 \\
34 \\
36\end{array}$ & $\begin{array}{r}11.0 \\
10.5 \\
10.5 \\
10.5 \\
10.5 \\
10.0 \\
10.0 \\
10.0 \\
10.0 \\
9.5 \\
8.5 \\
9.0 \\
8.5 \\
8.5 \\
8.0 \\
8.5 \\
8.0 \\
7.5\end{array}$ & $\begin{array}{l}2^{\prime \prime} \\
2^{\prime \prime} \\
2^{\prime \prime} \\
2^{\prime \prime} \\
2^{\prime \prime} \\
3^{\prime \prime} \\
3^{\prime \prime} \\
4^{\prime \prime} \\
4^{\prime \prime} \\
4^{\prime \prime} \\
4^{\prime \prime} \\
4^{\prime \prime} \\
4^{\prime \prime} \\
4^{\prime \prime} \\
5^{\prime \prime} \\
5^{\prime \prime} \\
6^{\prime \prime} \\
6^{\prime \prime}\end{array}$ & $\begin{array}{l}2^{\prime \prime} \\
2^{\prime \prime} \\
2^{\prime \prime} \\
2^{\prime \prime} \\
2^{\prime \prime} \\
2^{\prime \prime} \\
2^{\prime \prime} \\
2^{\prime \prime} \\
2^{\prime \prime} \\
2^{\prime \prime} \\
2^{\prime \prime} \\
2^{\prime \prime} \\
2^{\prime \prime}\end{array}$ & $\begin{array}{l}\text { Paresis } \\
\text { Death }\end{array}$ \\
\hline
\end{tabular}

* Cf. the foot note to No. 8. 
in question was 4 or 5 or even 6 seconds. After treating the blood specimens with ether, the shortest peroxidase stain-time became shorter than before.

No. 11

\begin{tabular}{|c|c|c|c|c|c|}
\hline \multirow{2}{*}{\multicolumn{2}{|c|}{$\begin{array}{c}\text { Day of experiment } \\
\text { (Date) }\end{array}$}} & \multirow{2}{*}{$\begin{array}{l}\text { Body } \\
\text { weight in } \\
\text { grms. }\end{array}$} & \multicolumn{2}{|c|}{$\begin{array}{c}\text { Shortest peroxidase } \\
\text { stain-time }\end{array}$} & \multirow[b]{2}{*}{ Remarks } \\
\hline & & & Fresh film & $\begin{array}{c}\text { After } \\
\text { treating* } \\
\text { with ether }\end{array}$ & \\
\hline \multicolumn{2}{|c|}{$\begin{array}{c}\text { Before the exp. } \\
(13 / I I)\end{array}$} & 17.5 & $2^{\prime \prime}$ & & \\
\hline $\begin{array}{c}\text { Days } \\
\text { of } \\
\text { feeding }\end{array}$ & $\begin{array}{l}2(15 . \mathrm{II}) \\
4 \\
6 \\
8 \\
10 \\
12 \\
14 \\
16 \\
18 \\
20 \\
22 \\
24 \\
25\end{array}$ & $\begin{array}{l}18.0 \\
18.0 \\
18.5 \\
18.5 \\
17.5 \\
17.5 \\
17.5 \\
16.5 \\
17.0 \\
17.0 \\
15.5 \\
13.5\end{array}$ & $\begin{array}{l}2^{\prime \prime} \\
2^{\prime \prime} \\
2^{\prime \prime} \\
2^{\prime \prime} \\
3^{\prime \prime} \\
3^{\prime \prime} \\
3^{\prime \prime} \\
4^{\prime \prime} \\
5^{\prime \prime} \\
5^{\prime \prime} \\
5^{\prime \prime} \\
5^{\prime \prime}\end{array}$ & $\begin{array}{l}2^{\prime \prime} \\
2^{\prime \prime} \\
2^{\prime \prime} \\
2^{\prime \prime} \\
2^{\prime \prime} \\
2^{\prime \prime} \\
2^{\prime \prime} \\
2^{\prime \prime}\end{array}$ & $\begin{array}{l}\text { Diarrhoea } \\
\text { Death }\end{array}$ \\
\hline
\end{tabular}

* Cf. the foot note to No. 8 .

No. 12

\begin{tabular}{|c|c|c|c|c|c|}
\hline \multirow{2}{*}{\multicolumn{2}{|c|}{$\begin{array}{c}\text { Day of experiment } \\
\text { (Date) }\end{array}$}} & \multirow{2}{*}{$\begin{array}{l}\text { Body } \\
\text { weight in } \\
\text { grms. }\end{array}$} & \multicolumn{2}{|c|}{$\begin{array}{l}\text { Shortest peroxidase } \\
\text { stain-time }\end{array}$} & \multirow[b]{2}{*}{ Remarks } \\
\hline & & & Fresh film & $\begin{array}{c}\text { After } \\
\text { treating* } \\
\text { with ether }\end{array}$ & \\
\hline \multicolumn{2}{|c|}{$\begin{array}{c}\text { Before the exp. } \\
(13 / \mathrm{II})\end{array}$} & 15.5 & $2^{\prime \prime}$ & & \\
\hline $\begin{array}{c}\text { Days } \\
\text { of } \\
\text { feeding }\end{array}$ & $\begin{array}{l}2(15 . I I) \\
4 \\
6 \\
8 \\
10 \\
12 \\
14 \\
16 \\
18 \\
20 \\
22 \\
24 \\
26 \\
28 \\
30 \\
32 \\
34 \\
36\end{array}$ & $\begin{array}{l}13.0 \\
12.5 \\
13.5 \\
13.5 \\
13.5 \\
13.5 \\
13.0 \\
12.5 \\
12.5 \\
12.5 \\
12.0 \\
11.5 \\
11.5 \\
12.0 \\
11.0 \\
10.5 \\
11.0 \\
10.0\end{array}$ & $\begin{array}{l}2^{\prime \prime} \\
2^{\prime \prime} \\
2^{\prime \prime} \\
2^{\prime \prime} \\
2^{\prime \prime} \\
3^{\prime \prime} \\
3^{\prime \prime} \\
3^{\prime \prime} \\
4^{\prime \prime} \\
4^{\prime \prime} \\
4^{\prime \prime} \\
4^{\prime \prime} \\
4^{\prime \prime} \\
4^{\prime \prime} \\
5^{\prime \prime} \\
5^{\prime \prime} \\
5^{\prime \prime} \\
5^{\prime \prime}\end{array}$ & $\begin{array}{l}2^{\prime \prime} \\
2^{\prime \prime} \\
2^{\prime \prime} \\
2^{\prime \prime} \\
2^{\prime \prime} \\
2^{\prime \prime} \\
2^{\prime \prime} \\
2^{\prime \prime} \\
2^{\prime \prime} \\
2^{\prime \prime} \\
2^{\prime \prime} \\
2^{\prime \prime} \\
2^{\prime \prime}\end{array}$ & $\begin{array}{l}\text { Slight paresis } \\
\text { Gait difficult, death in } \\
\text { the evening }\end{array}$ \\
\hline
\end{tabular}

* Cf. the foot note to No. 8. 
No. 13

\begin{tabular}{|c|c|c|c|c|c|}
\hline \multirow{2}{*}{\multicolumn{2}{|c|}{$\begin{array}{c}\text { Day of experiment } \\
\text { (Date) }\end{array}$}} & \multirow{2}{*}{$\begin{array}{l}\text { Body } \\
\text { weight in } \\
\text { grms. }\end{array}$} & \multicolumn{2}{|c|}{$\begin{array}{l}\text { Shortest peroxidase } \\
\text { stain-time }\end{array}$} & \multirow[b]{2}{*}{ Remarks } \\
\hline & & & Fresh film & $\begin{array}{c}\text { After } \\
\text { treating* } \\
\text { with ether }\end{array}$ & \\
\hline $\begin{array}{r}\text { Befor } \\
\quad \\
\end{array}$ & $\begin{array}{l}\text { the exp. } \\
\text { (II) }\end{array}$ & 15.5 & $2^{\prime \prime}$ & & \\
\hline $\begin{array}{c}\text { Days } \\
\text { of } \\
\text { feeding }\end{array}$ & $\begin{array}{l}2(15 . \mathrm{II}) \\
4 \\
6 \\
8 \\
10 \\
12 \\
14 \\
16 \\
18 \\
20 \\
22 \\
24 \\
26 \\
28 \\
30 \\
32 \\
34 \\
36 \\
38 \\
40 \\
42 \\
44 \\
46 \\
48 \\
50 \\
52 \\
54 \\
55\end{array}$ & $\begin{array}{l}15.5 \\
15.0 \\
15.0 \\
13.5 \\
14.0 \\
14.5 \\
14.0 \\
13.5 \\
14.0 \\
13.5 \\
13.0 \\
13.0 \\
13.0 \\
13.5 \\
13.0 \\
13.0 \\
12.5 \\
12.0 \\
12.5 \\
12.0 \\
12.0 \\
12.0 \\
12.0 \\
11.5 \\
11.0 \\
10.5 \\
10.5\end{array}$ & $\begin{array}{c}2^{\prime \prime} \\
2^{\prime \prime} \\
2^{\prime \prime} \\
2^{\prime \prime} \\
2^{\prime \prime} \\
3^{\prime \prime} \\
3^{\prime \prime} \\
3^{\prime \prime} \\
4^{\prime \prime} \\
4^{\prime \prime} \\
4^{\prime \prime} \\
4^{\prime \prime} \\
4^{\prime \prime} \\
4^{\prime \prime} \\
5^{\prime \prime} \\
5^{\prime \prime} \\
5^{\prime \prime} \\
5^{\prime \prime} \\
6^{\prime \prime} \\
7^{\prime \prime} \\
7^{\prime \prime} \\
7^{\prime \prime} \\
8^{\prime \prime} \\
10^{\prime \prime} \\
11^{\prime \prime} \\
13^{\prime \prime} \\
13^{\prime \prime}\end{array}$ & $\begin{array}{l}2^{\prime \prime} \\
2^{\prime \prime} \\
2^{\prime \prime} \\
2^{\prime \prime} \\
2^{\prime \prime} \\
2^{\prime \prime} \\
2^{\prime \prime} \\
2^{\prime \prime} \\
2^{\prime \prime} \\
2^{\prime \prime} \\
2^{\prime \prime} \\
2^{\prime \prime} \\
2^{\prime \prime} \\
3^{\prime \prime} \\
3^{\prime \prime} \\
3^{\prime \prime} \\
3^{\prime \prime} \\
3^{\prime \prime} \\
4^{\prime \prime} \\
5^{\prime \prime} \\
6^{\prime \prime} \\
6^{\prime \prime}\end{array}$ & $\begin{array}{l}\text { Gait difficult } \\
\text { Death }\end{array}$ \\
\hline
\end{tabular}

* Cf. the foot note to No. 8 .

3. The third series of experiment (Cf. Table 3).

Mice were fed on thoroughly washed polished rice and injected with $3 \%$ ammonium chloride every second day. The animals in this experiment were thus in a state of absolute B-avitaminosis and suffered from an insufficiency of liver function (produced with ammonium chloride). The result is as shown in the following Table 3 (Cf. No. $14-$ No. 20 in Table 3 ).

The length of life of mice after the commencement of feeding with absolutely B-deficient food was $\mathbf{1 5 . 4}$ days on an average. A prolongation of the shortest peroxidase stain-time occurred on the 4th day of feeding in all the mice, earlier than any clinical symptom. When a clinical symptom occurred at all, then the prolongation in question was as long as 6 or 7 or even 8 seconds. After treating the blood 
smears with ether, the shortest peroxidase stain-time became shorter than before.

4. The fourth series of experiment (Cf. Table 4).

Mice were fed on unpolished wheat with the aim of prevention of $\mathrm{B}$-avitaminosis, and all mice were injected with $3 \%$ ammonium chloride every second day, because the present experiment was performed for the purpose of investigating any influence of liver insuffciency upon the short-timed peroxidase reaction of blood leucocytes.

\section{TABle 3.}

Influence of liver insufficiency (produced with ammonium chloride) on the short-timed peroxidase reaction of leucocytes and symptoms of mice in the case of feeding with thoroughly washed

No. 14 polished rice. (No.14-No. 20)

\begin{tabular}{|c|c|c|c|c|c|c|}
\hline \multirow{2}{*}{\multicolumn{2}{|c|}{$\begin{array}{c}\text { Day of experiment } \\
\text { (Date) }\end{array}$}} & \multirow{2}{*}{$\begin{array}{c}\text { Body } \\
\text { weight } \\
\text { in grms. }\end{array}$} & \multirow{2}{*}{$\begin{array}{c}\text { Injection } \\
\text { of } 3 \% \\
\text { chlor- } \\
\text { ammonium } \\
\text { (c.c.) }\end{array}$} & \multicolumn{2}{|c|}{$\begin{array}{c}\text { Shortest peroxidase } \\
\text { stain-time }\end{array}$} & \multirow[b]{2}{*}{ Remarks } \\
\hline & & & & Fresh film & $\begin{array}{c}\text { After } \\
\text { treating* } \\
\text { with ether }\end{array}$ & \\
\hline \multicolumn{2}{|c|}{$\begin{array}{c}\text { Before the exp. } \\
(22 / \text { III })\end{array}$} & 10.5 & 0.1 & $2^{\prime \prime}$ & & \\
\hline $\begin{array}{l}\text { Days } \\
\text { of } \\
\text { feeding }\end{array}$ & $\begin{array}{l}2(25 . \mathrm{III}) \\
4 \\
6 \\
8 \\
9\end{array}$ & $\begin{array}{r}10.0 \\
11.0 \\
10.0 \\
8.0\end{array}$ & $\begin{array}{l}0.1 \\
0.1 \\
0.1 \\
0.08\end{array}$ & $\begin{array}{l}2^{\prime \prime} \\
3^{\prime \prime} \\
5^{\prime \prime} \\
7^{\prime \prime}\end{array}$ & $\begin{array}{l}2^{\prime \prime} \\
2^{\prime \prime} \\
2^{\prime \prime}\end{array}$ & $\begin{array}{l}\text { Diarrhoea } \\
\text { Death }\end{array}$ \\
\hline
\end{tabular}

* The shortest peroxidase stain-time of leucocytes on a film which had been treated with ether.

No. 15

\begin{tabular}{|c|c|c|c|c|c|c|}
\hline \multirow{2}{*}{\multicolumn{2}{|c|}{$\begin{array}{c}\text { Day of experiment } \\
\text { (Date) }\end{array}$}} & \multirow{2}{*}{$\begin{array}{c}\text { Body } \\
\text { weight } \\
\text { in grms. }\end{array}$} & \multirow{2}{*}{$\begin{array}{c}\text { Injection } \\
\text { of } 3 \% \\
\text { chlor- } \\
\text { ammonium } \\
\text { (c.c.) }\end{array}$} & \multicolumn{2}{|c|}{$\begin{array}{l}\text { Shortest peroxidase } \\
\text { stain-time }\end{array}$} & \multirow[b]{2}{*}{ Remarks } \\
\hline & & & & Fresh film & $\begin{array}{c}\text { After } \\
\text { treating* } \\
\text { with ether }\end{array}$ & \\
\hline \multicolumn{2}{|c|}{$\begin{array}{l}\text { Before the exp. } \\
(22 / \text { III })\end{array}$} & 10.5 & 0.1 & $2^{\prime \prime}$ & & \\
\hline $\begin{array}{l}\text { Days } \\
\text { of } \\
\text { feeding }\end{array}$ & $\begin{array}{l}2(25 . \mathrm{III}) \\
4 \\
6 \\
8 \\
10 \\
12 \\
14 \\
16\end{array}$ & $\begin{array}{r}11.5 \\
8.5 \\
8.0 \\
7.5 \\
8.5 \\
8.0 \\
7.5 \\
7.5\end{array}$ & $\begin{array}{l}0.11 \\
0.08 \\
0.08 \\
0.07 \\
0.08 \\
0.08 \\
0.07 \\
0.07\end{array}$ & $\begin{array}{r}8^{\prime \prime} \\
3^{\prime \prime} \\
5^{\prime \prime} \\
6^{\prime \prime} \\
8^{\prime \prime} \\
9^{\prime \prime} \\
10^{\prime \prime} \\
11^{\prime \prime}\end{array}$ & $\begin{array}{l}2^{\prime \prime} \\
2^{\prime \prime} \\
2^{\prime \prime} \\
3^{\prime \prime} \\
4^{\prime \prime} \\
4^{\prime \prime} \\
4^{\prime \prime}\end{array}$ & $\begin{array}{l}\text { Death soon after the } \\
\text { injection }\end{array}$ \\
\hline
\end{tabular}

* Cf. the foot note to No. 14 . 
No. 16

\begin{tabular}{|c|c|c|c|c|c|c|}
\hline \multirow{2}{*}{\multicolumn{2}{|c|}{$\begin{array}{c}\text { Day of experiment } \\
\text { (Date) }\end{array}$}} & \multirow{2}{*}{$\begin{array}{c}\text { Body } \\
\text { weight } \\
\text { in grms. }\end{array}$} & \multirow{2}{*}{\begin{tabular}{|c|} 
Injection \\
of $3 \%$ \\
chlor- \\
ammonium \\
(c.c.)
\end{tabular}} & \multicolumn{2}{|c|}{$\begin{array}{c}\text { Shortest peroxidase } \\
\text { stain-time }\end{array}$} & \multirow[b]{2}{*}{ Remarks } \\
\hline & & & & Fresh film & $\begin{array}{c}\text { After } \\
\text { treating* } \\
\text { with ether }\end{array}$ & \\
\hline \multicolumn{2}{|c|}{$\begin{array}{c}\text { Before the exp. } \\
(22 / \mathrm{III})\end{array}$} & 12.5 & 0.12 & $2^{\prime \prime}$ & & \\
\hline $\begin{array}{c}\text { Days } \\
\text { of } \\
\text { feeding }\end{array}$ & $\begin{array}{c}2(25 . \mathrm{III}) \\
4 \\
6 \\
8 \\
10 \\
12 \\
14 \\
16 \\
18 \\
20 \\
21\end{array}$ & $\begin{array}{r}11.5 \\
12.0 \\
11.5 \\
10.5 \\
10.5 \\
10.5 \\
9.5 \\
9.0 \\
9.0 \\
7.5 \\
7.0\end{array}$ & $\begin{array}{l}0.11 \\
0.11 \\
0.11 \\
0.1 \\
0.1 \\
0.1 \\
0.09 \\
0.09 \\
0.09 \\
0.07\end{array}$ & $\begin{array}{c}2^{\prime \prime} \\
4^{\prime \prime} \\
5^{\prime \prime} \\
6^{\prime \prime} \\
7^{\prime \prime} \\
7^{\prime \prime} \\
8^{\prime \prime} \\
9^{\prime \prime} \\
9^{\prime \prime} \\
10^{\prime \prime} \\
11^{\prime \prime}\end{array}$ & $\begin{array}{l}2^{\prime \prime} \\
2^{\prime \prime} \\
2^{\prime \prime} \\
2^{\prime \prime} \\
3^{\prime \prime} \\
3^{\prime \prime} \\
4^{\prime \prime} \\
5^{\prime \prime} \\
5^{\prime \prime} \\
5^{\prime \prime}\end{array}$ & $\begin{array}{l}\text { Convulsion after } \\
\text { the injection } \\
\text { Diarrhoea }\end{array}$ \\
\hline
\end{tabular}

* Cf. the foot note to No. 14.

No. 17

\begin{tabular}{|c|c|c|c|c|c|c|}
\hline \multirow{2}{*}{\multicolumn{2}{|c|}{$\begin{array}{c}\text { Day of experiment } \\
\text { (Date) }\end{array}$}} & \multirow{2}{*}{$\begin{array}{c}\text { Body } \\
\text { weight } \\
\text { in grms. }\end{array}$} & \multirow{2}{*}{$\begin{array}{l}\text { Injection } \\
\text { of } 3 \% \\
\text { chlor- } \\
\text { ammonium } \\
\text { (c.c.) }\end{array}$} & \multicolumn{2}{|c|}{$\begin{array}{c}\text { Shortest peroxidase } \\
\text { stain-time }\end{array}$} & \multirow[b]{2}{*}{ Remarks } \\
\hline & & & & Fresh film & $\begin{array}{c}\text { After } \\
\text { treating* } \\
\text { with ether }\end{array}$ & \\
\hline \multicolumn{2}{|c|}{$\begin{array}{l}\text { Before the exp. } \\
(22 / \mathrm{III})\end{array}$} & 15.5 & 0.15 & $\mathbf{2}^{\prime \prime}$ & & \\
\hline $\begin{array}{c}\text { Days } \\
\text { of } \\
\text { feeding }\end{array}$ & $\begin{array}{l}2 \text { (25. III) } \\
4 \\
6 \\
8 \\
10 \\
12 \\
14 \\
16 \\
18 \\
20 \\
21\end{array}$ & $\begin{array}{l}16.0 \\
16.0 \\
15.0 \\
14.0 \\
14.5 \\
14.0 \\
13.0 \\
13.0 \\
12.0 \\
11.0 \\
11.0\end{array}$ & $\begin{array}{l}0.16 \\
0.16 \\
0.15 \\
0.14 \\
0.14 \\
0.14 \\
0.13 \\
0.13 \\
0.12 \\
0.1\end{array}$ & $\begin{array}{r}2^{\prime \prime} \\
3^{\prime \prime} \\
4^{\prime \prime} \\
6^{\prime \prime} \\
7^{\prime \prime} \\
8^{\prime \prime} \\
8^{\prime \prime} \\
9^{\prime \prime} \\
9^{\prime \prime} \\
10^{\prime \prime} \\
10^{\prime \prime}\end{array}$ & $\begin{array}{l}2^{\prime \prime} \\
2^{\prime \prime} \\
2^{\prime \prime} \\
2^{\prime \prime} \\
3^{\prime \prime} \\
3^{\prime \prime} \\
3^{\prime \prime} \\
3^{\prime \prime} \\
4^{\prime \prime} \\
4^{\prime \prime}\end{array}$ & $\begin{array}{l}\text { Paresis } \\
\text { Death }\end{array}$ \\
\hline
\end{tabular}

* Cf. the foot note to No. 14.

The result will be seen from the following Table 4 (Cf. No. $21-N o .25$ in Table 4).

The shortest peroxidase stain-time was not prolonged in any of the cases, and any clinical symptom due to B-avitaminosis was never seen in any case. Body weight increased in all mice in spite of liver insufficiency, and not a single one of those mice died even after 30 days of the experiment. 
No. 18

\begin{tabular}{|c|c|c|c|c|c|c|}
\hline \multirow{2}{*}{\multicolumn{2}{|c|}{$\begin{array}{c}\text { Day of experiment } \\
\text { (Date) }\end{array}$}} & \multirow{2}{*}{$\begin{array}{c}\text { Body } \\
\text { weight } \\
\text { in grms. }\end{array}$} & \multirow{2}{*}{$\begin{array}{c}\text { Injection } \\
\text { of } 3 \% \\
\text { chlor- } \\
\text { ammonium } \\
\text { (c.c.) }\end{array}$} & \multicolumn{2}{|c|}{$\begin{array}{l}\text { Shortest peroxidase } \\
\text { stain-time }\end{array}$} & \multirow[b]{2}{*}{ Remarks } \\
\hline & & & & Fresh film & $\begin{array}{c}\text { After } \\
\text { treating* } \\
\text { with ether }\end{array}$ & \\
\hline \multicolumn{2}{|c|}{$\begin{array}{c}\text { Before the exp. } \\
(4 / \mathrm{IV})\end{array}$} & 12.5 & 0.12 & $2^{\prime \prime}$ & & \\
\hline $\begin{array}{l}\text { Days } \\
\text { of } \\
\text { feeding }\end{array}$ & $\begin{array}{r}2(6 . I V) \\
4 \\
6 \\
8 \\
10 \\
11\end{array}$ & $\begin{array}{r}11.0 \\
10.5 \\
11.0 \\
10.0 \\
9.5 \\
9.0\end{array}$ & $\begin{array}{l}0.11 \\
0.10 \\
0.11 \\
0.10 \\
0.09\end{array}$ & $\begin{array}{l}2^{\prime \prime} \\
4^{\prime \prime} \\
5^{\prime \prime} \\
6^{\prime \prime} \\
8^{\prime \prime} \\
8^{\prime \prime}\end{array}$ & $\begin{array}{l}2^{\prime \prime} \\
2^{\prime \prime} \\
2^{\prime \prime} \\
3^{\prime \prime} \\
3^{\prime \prime}\end{array}$ & $\begin{array}{l}\text { Paresis } \\
\text { Death }\end{array}$ \\
\hline
\end{tabular}

* Cf. the foot note to No. 14.

No. 19

\begin{tabular}{|c|c|c|c|c|c|c|}
\hline \multirow{2}{*}{\multicolumn{2}{|c|}{$\begin{array}{l}\text { Day of experiment } \\
\text { (Date) }\end{array}$}} & \multirow{2}{*}{$\begin{array}{c}\text { Body } \\
\text { weight } \\
\text { in grms. }\end{array}$} & \multirow{2}{*}{$\begin{array}{c}\text { Injection } \\
\text { of } 3 \% \\
\text { chlor- } \\
\text { ammonium } \\
\text { (c.c.) }\end{array}$} & \multicolumn{2}{|c|}{$\begin{array}{l}\text { Shortest peroxidase } \\
\text { stain-time }\end{array}$} & \multirow[b]{2}{*}{ Remarks } \\
\hline & & & & Fresh film & $\begin{array}{c}\text { After } \\
\text { treating* } \\
\text { with ether }\end{array}$ & \\
\hline \multicolumn{2}{|c|}{$\begin{array}{l}\text { Before the exp. } \\
(4 / I V)\end{array}$} & 11.0 & 0.11 & $2^{\prime \prime}$ & & \\
\hline $\begin{array}{l}\text { Days } \\
\text { of } \\
\text { feeding }\end{array}$ & $\begin{array}{r}2(6 . \mathrm{IV}) \\
4 \\
6 \\
8 \\
10 \\
12 \\
14 \\
15\end{array}$ & $\begin{array}{r}11.0 \\
10.5 \\
10.5 \\
10.0 \\
9.5 \\
9.0 \\
9.0 \\
9.0\end{array}$ & $\begin{array}{l}0.11 \\
0.10 \\
0.10 \\
0.10 \\
0.09 \\
0.09 \\
0.09\end{array}$ & $\begin{array}{r}2^{\prime \prime} \\
3^{\prime \prime} \\
4^{\prime \prime} \\
6^{\prime \prime} \\
7^{\prime \prime} \\
8^{\prime \prime} \\
9^{\prime \prime} \\
10^{\prime \prime}\end{array}$ & $\begin{array}{l}2^{\prime \prime} \\
2^{\prime \prime} \\
2^{\prime \prime} \\
2^{\prime \prime} \\
3^{\prime \prime} \\
3^{\prime \prime} \\
4^{\prime \prime}\end{array}$ & $\begin{array}{l}\text { Paresis } \\
\text { Gait impossible, } \\
\text { death in the night }\end{array}$ \\
\hline
\end{tabular}

* Cf. the foot note to No. 14.

No. 20

\begin{tabular}{|c|c|c|c|c|c|c|}
\hline \multirow{2}{*}{\multicolumn{2}{|c|}{$\begin{array}{c}\text { Day of experiment } \\
\text { (Date) }\end{array}$}} & \multirow{2}{*}{$\begin{array}{c}\text { Body } \\
\text { weight } \\
\text { in grms. }\end{array}$} & \multirow{2}{*}{\begin{tabular}{|c|} 
Injection \\
of $3 \%$ \\
chlor- \\
ammonium \\
(c.c.)
\end{tabular}} & \multicolumn{2}{|c|}{$\begin{array}{l}\text { Shortest peroxidase } \\
\text { stain-time }\end{array}$} & \multirow[b]{2}{*}{ Remarks } \\
\hline & & & & Fresh film & $\begin{array}{c}\text { After } \\
\text { treating* } \\
\text { with ether }\end{array}$ & \\
\hline \multicolumn{2}{|c|}{$\begin{array}{c}\text { Before the exp. } \\
(4 / \mathrm{IV})\end{array}$} & 10.5 & 0.10 & $2^{\prime \prime}$ & & \\
\hline $\begin{array}{c}\text { Days } \\
\text { of } \\
\text { feeding }\end{array}$ & $\begin{array}{l}2(6: I V) \\
4 \\
6 \\
8 \\
10 \\
12 \\
14 \\
15\end{array}$ & $\begin{array}{r}10.0 \\
10.5 \\
10.5 \\
10.0 \\
9.0 \\
9.0 \\
9.0 \\
9.0\end{array}$ & $\begin{array}{l}0.10 \\
0.10 \\
0.10 \\
0.10 \\
0.09 \\
0.09 \\
0.09\end{array}$ & $\begin{array}{c}2^{\prime \prime} \\
3^{\prime \prime} \\
4^{\prime \prime} \\
5^{\prime \prime} \\
6^{\prime \prime} \\
7^{\prime \prime} \\
9^{\prime \prime} \\
11^{\prime \prime}\end{array}$ & $\begin{array}{l}2^{\prime \prime} \\
2^{\prime \prime} \\
2^{\prime \prime} \\
2^{\prime \prime} \\
2^{\prime \prime} \\
3^{\prime \prime} \\
4^{\prime \prime}\end{array}$ & $\begin{array}{l}\text { Paresis } \\
\text { Gait impossible, } \\
\text { death in the night }\end{array}$ \\
\hline
\end{tabular}

* Cf. the foot note to No. 14 . 
TABLE 4.

Influenee of liver insufficiency (produced with ammonium ehloride) on the short-timed peroxidase reaction of leucocytes of mice in the case of feeding with not polished wheat. (No. 21-No. 25)

No. 21

\begin{tabular}{|c|c|c|c|c|}
\hline \multicolumn{2}{|c|}{$\begin{array}{l}\text { Day of } \\
\text { experiment } \\
\text { (Date) }\end{array}$} & 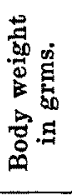 & 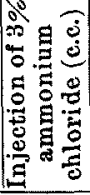 & 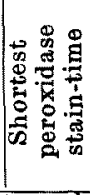 \\
\hline & $\begin{array}{l}\text { Before the exp. } \\
\text { (21/VII) }\end{array}$ & 9.0 & 0.09 & $2^{\prime \prime}$ \\
\hline 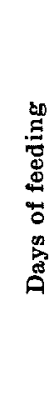 & $\begin{aligned} & 2(23 . \mathrm{VII}) \\
& 4 \\
& 6 \\
& 8 \\
& 10 \\
& 12 \\
& 14 \\
& 16 \\
& 18 \\
& 20 \\
& 22 \\
& 24 \\
& 26 \\
& 28 \\
& 30\end{aligned}$ & $\begin{array}{r}9.5 \\
9.5 \\
9.0 \\
10.0 \\
9.5 \\
10.0 \\
10.5 \\
12.0 \\
12.5 \\
13.5 \\
14.5 \\
14.5 \\
14.0 \\
15.0 \\
15.0\end{array}$ & $\begin{array}{l}0.09 \\
0.09 \\
0.09 \\
0.1 \\
0.09 \\
0.1 \\
0.1 \\
0.12 \\
0.12 \\
0.13 \\
0,14 \\
0.14 \\
0.14 \\
0.15\end{array}$ & $\begin{array}{l}2^{\prime \prime} \\
2^{\prime \prime} \\
2^{\prime \prime} \\
2^{\prime \prime} \\
2^{\prime \prime} \\
2^{\prime \prime} \\
2^{\prime \prime} \\
2^{\prime \prime} \\
2^{\prime \prime} \\
2^{\prime \prime} \\
2^{\prime \prime} \\
2^{\prime \prime} \\
2^{\prime \prime} \\
2^{\prime \prime} \\
2^{\prime \prime}\end{array}$ \\
\hline
\end{tabular}

No. 22

\begin{tabular}{|c|c|c|c|c|}
\hline & $\begin{array}{l}\text { Day of } \\
\text { experiment } \\
\text { (Date) }\end{array}$ & 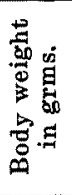 & 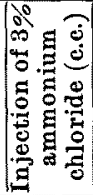 & 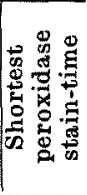 \\
\hline & $\begin{array}{l}\text { efore the exp. } \\
\text { (21/VII) }\end{array}$ & 12.0 & 0.12 & $2^{\prime \prime}$ \\
\hline 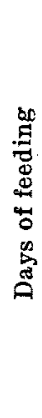 & $\begin{aligned} 2 & (23 . \mathrm{VII}) \\
4 & \\
6 & \\
8 & \\
10 & \\
12 & \\
14 & \\
16 & \\
18 & \\
20 & \\
22 & \\
24 & \\
26 & \\
28 & \\
30 & \end{aligned}$ & $\begin{array}{l}12.5 \\
12.5 \\
13.5 \\
14.0 \\
15.5 \\
16.0 \\
17.5 \\
17.5 \\
19.0 \\
19.0 \\
20.0 \\
21.0 \\
20.0 \\
20.5 \\
20.5\end{array}$ & $\begin{array}{l}0.12 \\
0.12 \\
0.13 \\
0.14 \\
0.15 \\
0.16 \\
0.17 \\
0.17 \\
0.19 \\
0.19 \\
0.20 \\
0.21 \\
0.20 \\
0.20\end{array}$ & $\begin{array}{l}2^{\prime \prime} \\
2^{\prime \prime} \\
2^{\prime \prime} \\
2^{\prime \prime} \\
2^{\prime \prime} \\
2^{\prime \prime} \\
2^{\prime \prime} \\
2^{\prime \prime} \\
2^{\prime \prime} \\
2^{\prime \prime} \\
2^{\prime \prime} \\
2^{\prime \prime} \\
2^{\prime \prime} \\
2^{\prime \prime} \\
2^{\prime \prime}\end{array}$ \\
\hline
\end{tabular}

No. 23

\begin{tabular}{|c|c|c|c|c|}
\hline & $\begin{array}{l}\text { Day of } \\
\text { experiment } \\
\text { (Date) }\end{array}$ & 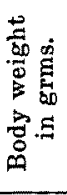 & 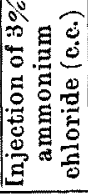 & 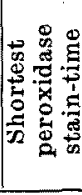 \\
\hline & $\begin{array}{l}\text { ore the exp. } \\
\text { (21/VII) }\end{array}$ & 11.0 & 0.11 & $2^{\prime \prime}$ \\
\hline 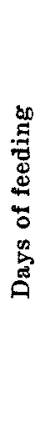 & $\begin{aligned} 2 & (23, \mathrm{VII}) \\
4 & \\
6 & \\
8 & \\
10 & \\
12 & \\
14 & \\
16 & \\
18 & \\
20 & \\
22 & \\
24 & \\
26 & \\
28 & \\
30 & \end{aligned}$ & $\begin{array}{l}10.5 \\
10.0 \\
10.5 \\
10.0 \\
10.0 \\
10.0 \\
10.5 \\
10.5 \\
12.0 \\
11.5 \\
13.0 \\
13.0 \\
12.0 \\
13.0 \\
13.0\end{array}$ & $\begin{array}{l}0.10 \\
0.10 \\
0.10 \\
0.10 \\
0.10 \\
0.10 \\
0.10 \\
0.10 \\
0.12 \\
0.11 \\
0.13 \\
0.13 \\
0.12 \\
0.13\end{array}$ & $\begin{array}{l}2^{\prime \prime} \\
2^{\prime \prime} \\
2^{\prime \prime} \\
2^{\prime \prime} \\
2^{\prime \prime} \\
2^{\prime \prime} \\
2^{\prime \prime} \\
2^{\prime \prime} \\
2^{\prime \prime} \\
2^{\prime \prime} \\
2^{\prime \prime} \\
2^{\prime \prime} \\
2^{\prime \prime} \\
2^{\prime \prime} \\
2^{\prime \prime}\end{array}$ \\
\hline
\end{tabular}

No. 24

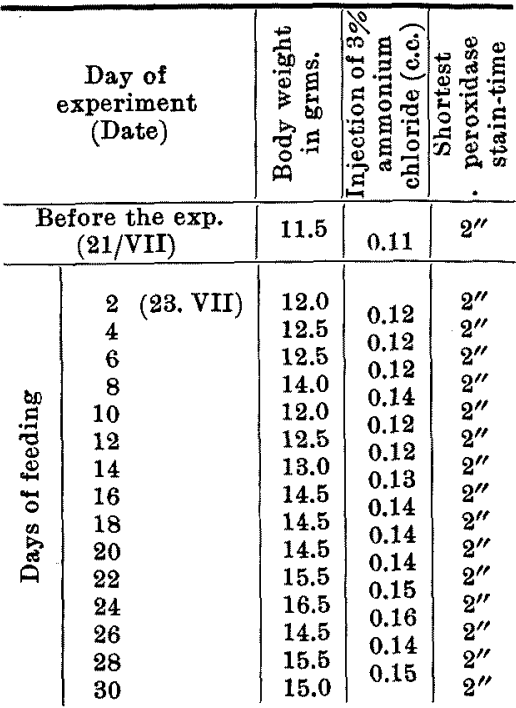

Experiment was stopped. Mouse was vigorous. 
No. 25

\begin{tabular}{|c|c|c|c|c|}
\hline \multicolumn{2}{|c|}{$\begin{array}{c}\text { Day of experiment } \\
\text { (Date) }\end{array}$} & $\begin{array}{l}\text { Body weight } \\
\text { in grms. }\end{array}$ & $\begin{array}{l}\text { Injection of } 3 \% \\
\text { ammonium } \\
\text { chloride (c. c.) }\end{array}$ & $\begin{array}{c}\text { Shortest peroxidase } \\
\text { stain-time }\end{array}$ \\
\hline \multicolumn{2}{|c|}{$\begin{array}{c}\text { Before the exp. } \\
(21 / \text { VII })\end{array}$} & 13.0 & 0.13 & $2^{\prime \prime}$ \\
\hline $\begin{array}{l}\text { Days } \\
\text { of } \\
\text { feeding }\end{array}$ & $\begin{aligned} 2 & (23 . \mathrm{VII}) \\
4 & \\
6 & \\
8 & \\
10 & \\
12 & \\
14 & \\
16 & \\
18 & \\
20 & \\
22 & \\
24 & \\
26 & \\
28 & \\
30 & \end{aligned}$ & $\begin{array}{l}12.5 \\
12.5 \\
13.0 \\
14.0 \\
13.0 \\
12.5 \\
12.5 \\
14.0 \\
14.5 \\
14.0 \\
15.0 \\
16.0 \\
15.0 \\
16.0 \\
16.0\end{array}$ & $\begin{array}{l}0.12 \\
0.12 \\
0.13 \\
0.14 \\
0.13 \\
0.12 \\
0.12 \\
0.14 \\
0.14 \\
0.14 \\
0.15 \\
0.16 \\
0.15 \\
0.16\end{array}$ & $\begin{array}{l}2^{\prime \prime} \\
2^{\prime \prime} \\
2^{\prime \prime} \\
2^{\prime \prime} \\
2^{\prime \prime} \\
2^{\prime \prime} \\
2^{\prime \prime} \\
2^{\prime \prime} \\
2^{\prime \prime} \\
2^{\prime \prime} \\
2^{\prime \prime} \\
2^{\prime \prime} \\
2^{\prime \prime} \\
2^{\prime \prime} \\
2^{\prime \prime}\end{array}$ \\
\hline
\end{tabular}

Experiment was stopped. Mouse was vigorous.

\section{Comment.}

1. Length of life of mice after feeding with polished rice (Cf. Table 5).

TABLE 5.

Length of life after feeding with polished rice.

\begin{tabular}{|c|c|c|c|c|c|c|}
\hline \multirow[b]{2}{*}{$\begin{array}{l}\text { Length of life in } \\
\text { days (No. of } \\
\text { cases) }\end{array}$} & \multicolumn{2}{|c|}{$\begin{array}{l}\text { I. Case of feeding } \\
\text { with thoroughly } \\
\text { washed rice }\end{array}$} & \multicolumn{2}{|c|}{$\begin{array}{l}\text { II. Case of feeding } \\
\text { with not washed } \\
\text { rice }\end{array}$} & \multicolumn{2}{|c|}{$\begin{array}{l}\text { III. Case of feeding } \\
\text { with thoroughly } \\
\text { washed rice, with an } \\
\text { injection of } 3 \% \mathrm{NH}_{4} \mathrm{Cl}\end{array}$} \\
\hline & $\begin{array}{l}19 \\
21 \\
22 \\
23 \\
24\end{array}$ & $\begin{array}{l}(2) \\
(1) \\
(2) \\
(1) \\
(1)\end{array}$ & $\begin{array}{l}25 \\
30 \\
34 \\
36 \\
55\end{array}$ & $\begin{array}{l}(1) \\
(1) \\
(1) \\
(2) \\
(1)\end{array}$ & $\begin{array}{r}9 \\
11 \\
15 \\
16 \\
21\end{array}$ & $\begin{array}{l}(1) \\
(1) \\
(2) \\
(1) \\
(2)\end{array}$ \\
\hline $\begin{array}{l}\text { Average length } \\
\text { of life in days }\end{array}$ & \multicolumn{2}{|c|}{21.4} & \multicolumn{2}{|c|}{36} & \multicolumn{2}{|c|}{15.4} \\
\hline
\end{tabular}

Among three groups of $\mathrm{B}$-avitaminotic mice, the group of the relatively $\mathrm{B}$-avitaminotic had the longest life ( 36 days on an average) and the length of life of the B-avitaminotic animals with liver insuffciency was the shortest (15.4 days on an average). The absolutely $\mathrm{B}$-avitaminotic mice generally lived as long as three weeks (21.4 days on an average). Thus, the ratio of lengths of life compared among 
those three groups of mice is as follows :-

$\begin{aligned} & \text { Relative } \\ & \text { B-avitaminosis }\end{aligned}: \begin{aligned} & \text { Absolute } \\ & \text { B-avitaminosis }\end{aligned}: \begin{aligned} & \text { Absolute B-avitaminosis } \\ & \text { with liver insufficiency }\end{aligned}=5: 3: 2$ Table 6).

2. Prolongation of the shortest Peroxidase stain-time (Cf.

TABLE 6.

Relation between prolongation of the shortest peroxidase stain-time and the length of days of feeding with polished rice.

\begin{tabular}{|c|c|c|c|c|c|c|}
\hline \multirow{3}{*}{$\begin{array}{c}\text { The } \\
\text { shortest } \\
\text { peroxidase } \\
\text { stain-time }\end{array}$} & \multicolumn{6}{|c|}{ Number of days of feeding with } \\
\hline & \multicolumn{2}{|c|}{$\begin{array}{l}\text { Thoroughly washed } \\
\text { rice }\end{array}$} & \multicolumn{2}{|c|}{ Not washed rice } & \multicolumn{2}{|c|}{$\begin{array}{c}\text { Thoroughly washed rice } \\
\text { and injection of } 3 \% \\
\text { chlor-ammonium }\end{array}$} \\
\hline & Days $\begin{array}{c}\text { No. of } \\
\text { cases) }\end{array}$ & Average & Days $\begin{array}{c}\text { No. of } \\
\text { cases) }\end{array}$ & Average & Days $\begin{array}{c}\text { No. of } \\
\text { cases }\end{array}$ & Average \\
\hline $3^{\prime \prime}$ & $\begin{array}{ll}4 & (2) \\
6 & (5)\end{array}$ & 5.4 & $\begin{array}{l}10 \\
12\end{array}$ & 11.3 & $4 \quad(5)$ & 4 \\
\hline $4^{\prime \prime}$ & $\begin{array}{rr}8 & (1) \\
10 & (4) \\
12 & (2)\end{array}$ & 10.3 & $\begin{array}{ll}16 & (3) \\
18 & (2) \\
22 & (1)\end{array}$ & 17.6 & $\begin{array}{ll}4 & (2) \\
6 & (3)\end{array}$ & 5.2 \\
\hline $5^{\prime \prime}$ & $\begin{array}{ll}12 & (3) \\
16 & (1)\end{array}$ & 13 & $\begin{array}{ll}18 & (1) \\
30 & (4) \\
32 & (1)\end{array}$ & 28.3 & $\begin{array}{ll}6 & (4) \\
8 & (1)\end{array}$ & 6.4 \\
\hline $6^{\prime \prime}$ & $\begin{array}{ll}12 & (1) \\
14 & (2) \\
16 & (2) \\
18 & (2) \\
\end{array}$ & 15.4 & $\begin{array}{ll}34 & (5) \\
38 & (1)\end{array}$ & 35.3 & $\begin{aligned} 8 & (5) \\
10 & (1)\end{aligned}$ & 8.3 \\
\hline $7^{\prime \prime}$ & $\begin{array}{ll}14 & (2) \\
16 & (1) \\
18 & (1) \\
20 & (3) \\
\end{array}$ & 17.4 & $40 \quad(1)$ & 40 & $\begin{array}{rr}8 & (1) \\
10 & (3) \\
12 & (1)\end{array}$ & 10 \\
\hline $8^{\prime \prime}$ & $\begin{array}{ll}16 & (1) \\
18 & (1) \\
22 & (1)\end{array}$ & 18.7 & $46 \quad(1)$ & 46 & $\begin{array}{ll}10 & (2) \\
12 & (2) \\
14 & (1)\end{array}$ & 11.6 \\
\hline $9^{\prime \prime}$ & $\begin{array}{ll}18 & (1) \\
21 & (1)\end{array}$ & 19.5 & - & - & $\begin{array}{ll}12 & (1) \\
14 & (2) \\
16 & (2)\end{array}$ & 14.4 \\
\hline $10^{\prime \prime}$ & $18 \quad(1)$ & 18 & $48 \quad(1)$ & 48 & $\begin{array}{ll}14 & (1) \\
15 & (1) \\
20 & (2)\end{array}$ & 17.2 \\
\hline $11^{\prime \prime}$ & $24 \quad(1)$ & 24 & (1) & 50 & $\begin{array}{ll}15 & (1) \\
16 & (1) \\
21 & (1)\end{array}$ & 17.3 \\
\hline $13^{\prime \prime}$ & $-\quad-$ & - & $52 \quad(1)$ & 52 & - & - \\
\hline
\end{tabular}


As is shown in Table 6, prolongation of the shortest peroxidase stain-time occurred most early in the case of absolutely B-avitaminotic mice with liver insufficiency. All of them showed it on the fourth day of experiment. The latest occurrence of the prolongation fell in the group of relatively B-avitaminotic mice (after averaging 11.3 days of feeding). The absolutely B-avitaminotic mice began to show the prolongation in question after averaging 5.4 days of feeding.

In the group of absolute B-avitaminosis with a liver insufficiency, the prolongation in question was 6 seconds after averaging 8.3 days of feeding and 8 seconds after averaging $\mathbf{1 1 . 6}$ days, so that the acceleration of the prolongation was the largest. The group of relative $\mathrm{B}$-avitaminosis showed 6 seconds time of the shortest peroxidase reaction after averaging 35.3 days of feeding, and 8 seconds time after averaging 46 days, so that the acceleration was the smallest. The group of absolute B-avitaminosis showed an intermediate relation. The shortest peroxidase stain-time was 6 seconds after averaging 15.4 days of feeding and 8 seconds after averaging 18.7 days.

Now, in the group of non-B-avitaminotic mice in the fourth series of experiment, the prolongation in question did not occur in spite of the fact that the same trial to cause a state of liver insufficiency was performed as in the case of the group of B-avitaminotic mice in the third series of experiment. This fact shows that the liver insufficiency produced with ammonium chloride in the amount used will not cause a prolongation of the shortest peroxidase stain-time at all. Thus, the earliest on set of the prolongation in question and the largest acceleration of it which occurred in the group of the absolutely B-avitaminotic mice with liver insufficiency are not due to a direct influence of the liver insufficiency upon the short-timed peroxidase reaction. The liver insufficiency produced with ammonium chloride may indeed intensify the injury resulting from $\mathrm{B}$-avitaminosis. It may be assumed that a certain ether-soluble substance ${ }^{5)}$ or substances, which are produced in a state of B-avitaminosis, will weaken the peroxidase reaction of blood leucocytes, at least as far as the short-timed peroxidase reaction is concerned, and will increase with a rather remarkable speed in a body with a liver insufficiency.

The rice disease of mice fed on absolutely B-deficient food and that of mice fed on relatively B-deficient food are, as have been stated

5) A. T a kamats u, Tohoku J. Exp. Med., 1934, 23, 46; T. S uzuki and A. Takam a t s u, Ibid., 1934, 24, 202 ; T. Suzuki, Ibid., 1935, 25, 186, 201 and 575 ; T. Suzuki and A. T a k a m at s u, Ibid., 1935, 25, 588. 
by Ogata and his collaboraters, identical, but the latter will survive the former much longer. And from the view point of the short-timed peroxidase reaction of blood leucocytes, the prolongation of it will occur remarkably earlier in the case of feeding with thoroughly washed rice than in the case of feeding with unwashed rice, so that some difference will be seen between them.

By the way, it has been shown in the present paper that a prolongation of the shortest peroxidase stain-time is a sign of avitaminosis $B$, and that it can not be caused by liver insufficiency produced by ammonium chloride.

\section{Conclusions.}

A prolongation of the shortest peroxidase stain-time of blood leucocytes was compared between absolute and relative B-avitaminosis, and further between B-avitaminosis with liver insufficiency. In the group of mice fed on absolutely B-avitaminotic food and put in a state of liver insufficiency, the prolongation in question began to occur most early and became marked most rapidly, and the average length of life was the shortest. A commencement and an increase of the prolongation in question occurred latest of all in the group of mice fed on relatively B-avitaminotic food, and the life of this group was the longest. The group of mice fed on absolutely B-avitaminotic food showed an intermediate relation. 